\title{
Experimental Study on Core Permittivity of Xinjiang Oilfield
}

\author{
Ke Shizhen ${ }^{1}$, Gao Jianying ${ }^{1}$, Sun Yanru ${ }^{1}$, Cai Minlong ${ }^{2}$ and Ma Runsheng ${ }^{2}$ \\ (1. Institute of Resource and Information, China University of Petroleum, Beijing 102249, China) \\ (2. Xijiang Well Logging Company, Karamay 834000, Xinjiang, China)
}

\begin{abstract}
This paper introduces the principles and procedures of the core permittivity measurement using a parallel capacitor with an electromagnetic shield. The cores from the Xinjiang oilfield were measured by using the frequency-swept method at different salinities and saturations. The effects of salinity, frequency, porosity and saturation on core permittivity were investigated. The relationship of core permittivity versus water saturation and the relationship of dry core permittivity versus porosity were obtained. The permittivity values of rock matrix and oil were obtained at a frequency of $47 \mathrm{MHz}$ and $200 \mathrm{MHz}$, and would provide guidance for the interpretation of dual frequency dielectric logging data.
\end{abstract}

Key words: Permittivity, measurement, frequency-swept, core, water saturation

\section{Introduction}

In petroleum prospecting, rock permittivity is widely used by engineers because of its large difference between oil and water and the small effect of changing salinity of formation water. Many researchers have also investigated core permittivity (Shang and Feng, 1995; Feng, et al., 1995; Xie, 2001). Although dielectric well logging has gradually phased out from the service market because of its small depth of investigation, dual frequency dielectric logging is still used in the Xinjiang oilfield, because it is better than the conventional resistivity logging, with which it is difficult to recognize oil and gas. The study of core permittivity of the Xinjiang oilfield would provide guidance for the interpretation of dual frequency dielectric logging data.

\section{Measurement principles}

There are many techniques for the measurement of rock permittivity, such as the coaxial scattering method (S parameter), resonant cavity method and parallel capacitor method. For the frequency range of $20-270 \mathrm{MHz}$, because this frequency range is sensitive to environment, the parallel capacitor with shield was selected for measurement. The system diagram is shown in Fig. 1.

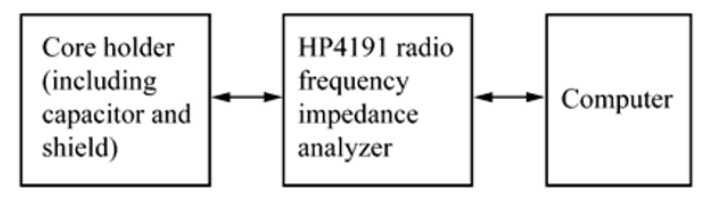

Fig. 1 Diagram of core permittivity measurement system using a parallel capacitor with a shield
The parallel capacitor with shield could be replaced by an equivalent of a $\pi$ electrical conductance network (as shown in Fig. 2).

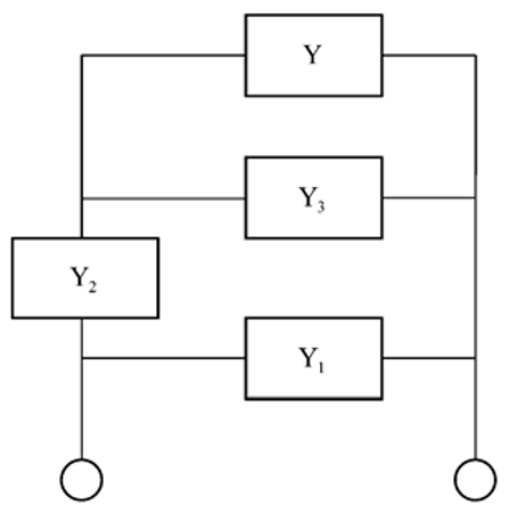

Fig. 2 Equivalent of $\pi$ electrical conductance network

Where, $Y_{1}, Y_{2}$ and $Y_{3}$ constitute the equivalent circuit of the shield. $Y$ is the conductance of the parallel capacitor and the measured sample. According to the equivalent network, the conductance obtained by the impedance analyzer can be expressed as follows:

$$
Y_{M}=Y_{1}+\frac{Y_{2} Y_{3}+Y_{2} Y}{Y_{2}+Y_{3}+Y}
$$

Before the measurement of the samples, three kinds of known samples, i.e., air, brass and plexiglass, were used to determine $Y_{1}, Y_{2}$ and $Y_{3}$. The conductance obtained by the impedance analyzer with the three known samples was respectively denoted by $Y_{M a}, Y_{M b}$ and $Y_{M \mathrm{p}}$, then

$$
Y_{M \mathrm{a}}=Y_{1}+\frac{Y_{2} Y_{3}+Y_{2} Y_{\mathrm{a}}}{Y_{2}+Y_{3}+Y_{\mathrm{a}}}
$$




$$
\begin{gathered}
Y_{M \mathrm{~b}}=Y_{1}+\frac{Y_{2} Y_{3}+Y_{2} Y_{\mathrm{b}}}{Y_{2}+Y_{3}+Y_{\mathrm{b}}} \\
Y_{M \mathrm{p}}=Y_{1}+\frac{Y_{2} Y_{3}+Y_{2} Y_{\mathrm{p}}}{Y_{2}+Y_{3}+Y_{\mathrm{P}}}
\end{gathered}
$$

where $Y_{\mathrm{a}}, Y_{\mathrm{b}}$ and $Y_{\mathrm{p}}$ are known.

The parameters $Y_{1}, Y_{2}$ and $Y_{3}$ can be obtained by solving the equations above. Thus the conductance of capacitor with core, $Y$, can be determined by using the measured conductance $Y_{M}$ through equation (1).

After calibration of the system, we can start to measure the core samples. When a core sample was measured, the conductance $Y_{M}$ was obtained by the impedance analyzer, and $Y$ was derived from $Y_{M}$ with Eq. (1), then the permittivity $\varepsilon$ and conductivity $\sigma$ of the measured sample were calculated with the following two sets of equations.

Static equation: $Y=G+j \omega C=\frac{A}{d}(\sigma+j \omega \varepsilon)$

$$
\left\{\begin{array}{c}
\varepsilon=\frac{d}{A} \omega \operatorname{Im}(Y) \\
\sigma=\frac{d}{A} \operatorname{Re}(Y)
\end{array}\right.
$$

Dynamic equation (Shang and Feng, 1995):

$$
\begin{gathered}
Y=\frac{\pi a^{2}}{d}(\sigma+j \omega C)=\frac{2 J_{1}(k a)}{k a J_{0}(k a)} \\
\sigma+j \omega \varepsilon=\frac{\frac{Y d}{\pi a^{2}}(k a) J_{0}(k a)}{2 J_{1}(k a)}
\end{gathered}
$$

where $k$ is wave-number vector; $\omega$ is angular frequency; $J_{0}$ and $J_{1}$ are respectively the zeroth-order and the first-order Bessel functions; $d$ is the thickness of sample; $a$ is the radius of sample; $A$ is the area of the capacitor plate. $G$ is conductance, and $C$ is capacitance.

\section{Measurement procedures}

\subsection{Preparation of sample}

The core was cut into small cylinders, and smoothed to make the top surface parallel with the bottom surface and perpendicular with the cylinder surface. The diameter of core cylinder was $38 \mathrm{~mm}$ and the thickness was $6 \mathrm{~mm}$. The thickness error should not exceed 0.2 $\mathrm{mm}$. After washing to oil-free and salt-free, the core cylinder was put in a dryer for use.

\subsection{Measurement of sample porosity}

The dry core sample was weighed with an electronic balance to obtain its dry weight, $W_{0}$, and then the core was saturated with brine and weighed again to get the wet weight of the sample, $W_{1}$. The pore volume of the sample is the difference of $W_{1}$ and $W_{0}$ divided by the density of brine. The bulk volume of the sample was obtained by measuring its radius and thickness. The porosity of the core sample is the ratio of pore volume to bulk volume.

\subsection{Saturation of samples with brine}

Brines with different salinities were prepared and put in a sealed vessel to prevent evaporation of moisture. The dry core sample was put into the brine with a specified salinity to be saturated. The procedure of saturation process was as follows: First the sample was put in a steel vessel, which was connected with a vacuum pump and vacuumized for 5-6 hours. Then the steel vessel was filled up with brine through a valve and vacuumized again for 5-6 hours to make sure the sample was completely saturated.

\subsection{Measurement of sample permittivity}

The saturated sample was put in the core holder and the conductance $Y_{M}$ of the whole holder was measured. The permittivity and conductivity of the saturated core sample was calculated from $Y_{M}$ by using equations (5) and (6). The measurement was repeated with core samples of different water saturations until the core dried out. The permittivity and conductivity of the core with different water saturations was measured in a frequency range of 20 $\mathrm{MHz}$ to $270 \mathrm{MHz}$.

\section{Measurement results}

Twenty cores from the Xinjiang oilfield were saturated with brines with salinities of 3,15 and 25 $\mathrm{kppm}$, and the permittivity values of the saturated samples were measured. The permittivity values of oil and brine were also measured separately. The permittivity values at frequencies of $47 \mathrm{MHz}$ and $200 \mathrm{MHz}$ were determined because they are used in dual frequency dielectric logging.

\subsection{Measurement result of brine permittivity}

The permittivity measurement results of three brines with salinities of 3, 15 and $25 \mathrm{kppm}$ are shown in Fig. 3. The results indicated that the frequency dispersion of low salinity brine was not obvious. The influence of frequency on permittivity was small for the brine with low salinity. 


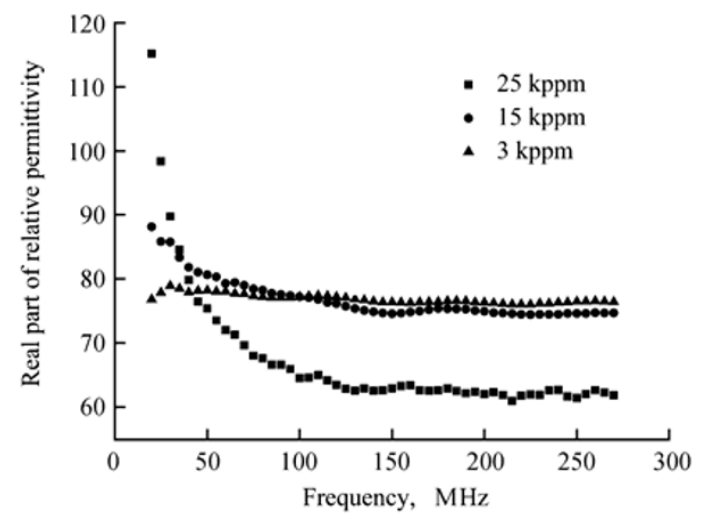

Fig. 3 Permittivity of brines with different salinities

\subsection{Measurement result of core permittivity}

The relationship of permittivity with frequency of 20 cores from the Xinjiang oilfield is shown in Fig. 4. It is seen that core permittivity decreased with increasing frequency, namely there existed frequency dispersion. Core permittivity increased with increasing water saturation of the core.

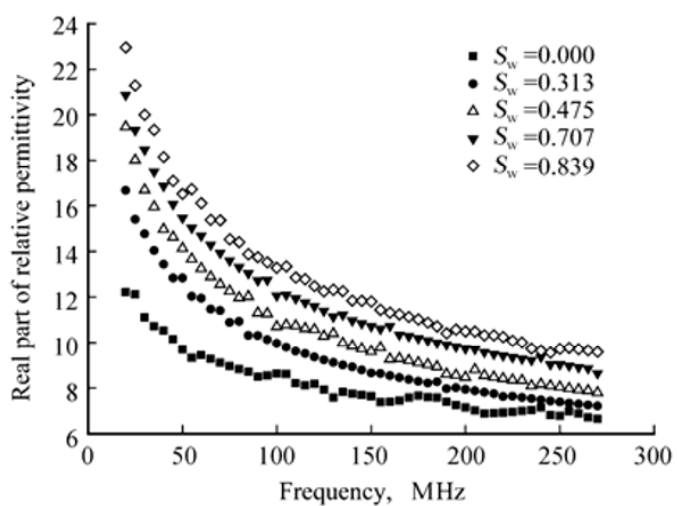

Fig. 4 Relationship of permittivity with frequency and water saturation

The relationship of rock permittivity with water saturation at a frequency of $47 \mathrm{MHz}$ or $200 \mathrm{MHz}$ is shown in Figs. 5-6.

It is seen from Figs. 5-6 that the rock permittivity had good linear relationship with $\phi S_{\mathrm{w}}$.

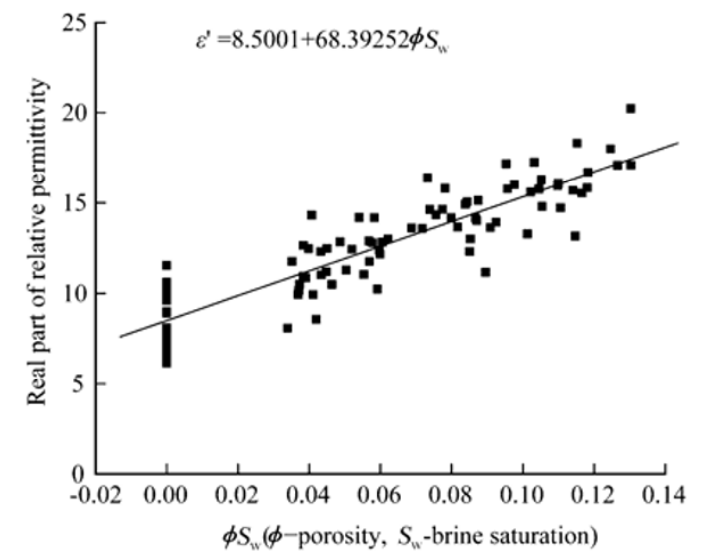

Fig. 5 Relationship of rock permittivity and $\left(\phi S_{\mathrm{w}}\right)$ at $47 \mathrm{MHz}$

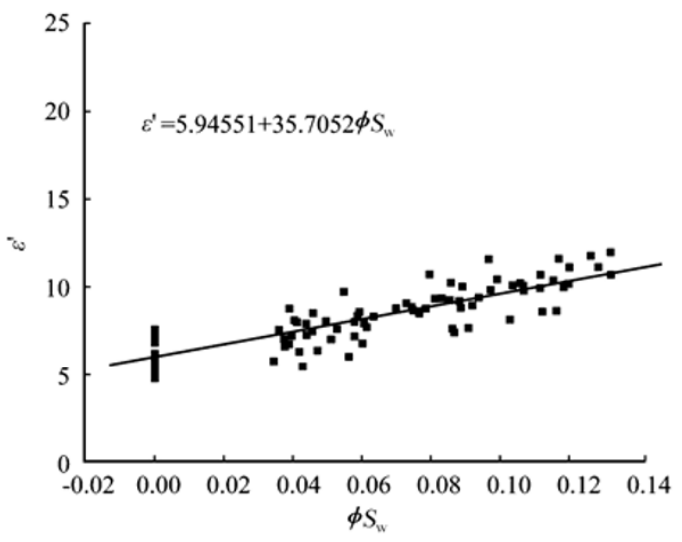

Fig. 6 Relationship of rock permittivity and $\phi S_{\mathrm{w}}$ at $200 \mathrm{MHz}$

\subsection{Influence of salinity on core permittivity}

Linear function $\varepsilon=\mathrm{A}+\mathrm{B}\left(\phi S_{\mathrm{w}}\right)$ was used to fit the relationship of the permittivity and the product of water saturation and porosity $\left(\phi S_{\mathrm{w}}\right)$, or named water volume, at different salinities, and the result in Table 1 was obtained.

Table 1 Linear function $=\mathbf{A}+\mathbf{B}\left(\phi S_{\mathrm{w}}\right)$ at different salinities

\begin{tabular}{cccc}
\hline Salinity, kppm & Frequency, MHz & Constant A & Slope B \\
\hline \multirow{2}{*}{3} & 47 & 7.8 & 49.6 \\
& 200 & 5.3 & 37.5 \\
\hline \multirow{2}{*}{8} & 47 & 8.5 & 68.4 \\
& 200 & 5.9 & 35.7 \\
\hline \multirow{2}{*}{15} & 47 & 8.3 & 88.3 \\
& 200 & 5.4 & 49.0 \\
\hline \multirow{2}{*}{25} & 47 & 8.3 & 102.3 \\
& 200 & 5.5 & 52.0 \\
\hline \multirow{2}{*}{30} & 47 & 8.4 & 92.5 \\
& 200 & 5.4 & 54.9 \\
\hline
\end{tabular}

Table 1 shows that the intercept A did not change obviously with the salinity of brine, while the slope B increased slightly and decreased when the salinity of brine is higher. The slope $\mathrm{B}$ at a low frequency decreased after $25 \mathrm{kppm}$, but the slope B at a high frequency kept increasing.

\subsection{Relationship of permittivity of dry core sample and porosity}

Through the analysis of 20 dry core samples, the relationship of permittivity and porosity at $47 \mathrm{MHz}$ and $200 \mathrm{MHz}$ was obtained as follows.

$47 \mathrm{MHz}$ :

$$
\mathcal{E}^{\prime}=9.83319-20.75633 \phi
$$

$200 \mathrm{MHz}:$

$$
\varepsilon^{\prime}=5.80454-6.66838 \phi
$$


where, $\varepsilon^{\prime}$ is the real part of relative permittivity; $\phi$ is the porosity of core.

In this way, we can obtain the permittivity of rock matrix, namely the permittivity of rock matrix when $\phi$ $=0$.

\section{Conclusion}

1) The permittivity of rock matrix, oil and water

According to the core experiment, the permittivity of rock matrix of the Xinjiang oilfield at $47 \mathrm{MHz}$ and $200 \mathrm{MHz}$ was 9.83 and 5.80, respectively. Oil permittivity was 2.5 at both $47 \mathrm{MHz}$ and $200 \mathrm{MHz}$. The permittivity of brine with a salinity lower than $15 \mathrm{kppm}$ was 80 at both $47 \mathrm{MHz}$ and $200 \mathrm{MHz}$, but that of brine with a salinity of $25 \mathrm{kppm}$ was 73 and 65 at $47 \mathrm{MHz}$ and $200 \mathrm{MHz}$, respectively.

2) Frequency dispersion

a) Permittivity decreased with increasing frequency. Permittivity decreased sharply with increasing frequency at a low frequency but changed gently at a high frequency.

b) The measurement of dry sample still had certain frequency dispersion, and this phenomenon was possibly due to the influence of immovable water or other ions in micro-pores of the core.

3) Relationship of rock permittivity with water volume $\left(\phi S_{\mathrm{w}}\right)$.

At $47 \mathrm{MHz}$ or $200 \mathrm{MHz}$, permittivity increased with increasing water volume $\left(\phi S_{\mathrm{w}}\right)$, and the change of permittivity with water volume was greater at $47 \mathrm{MHz}$ than that at $200 \mathrm{MHz}$.

4) Influence of salinity on permittivity

a) The permittivity of brine with a higher salinity was lower than that with a lower salinity. The influence of salinity on permittivity was small when salinity was smaller than $25 \mathrm{kppm}$, especially at a higher frequency.

b) Rock permittivity increased more significantly with increasing water volume $\left(\phi S_{\mathrm{w}}\right)$ for the brine with a higher salinity, namely the value of slope B in Table 1 increased with increasing salinity.

\section{Reference}

Feng Q. N., Li X. M. and Zheng H. H. (1995) Experimental study on rock dielectric permittivity at $1 \mathrm{kHz}-15 \mathrm{MHz}$. Geophysics Journal, 38 (supplement 1), 331-336 (in Chinese)

Shang Z. Y. and Feng Q. N. (1995) Rock dielectric permittivity measurement systems at three kinds of frequency bands and measurement results. Geophysics Journal, 38(supplement 1), 253-259 (in Chinese)

Xie R. H. (2001) Frequency dispersion of rock permittivity in radio frequency range. Journal of University of Petroleum, 25 (1), 90-92 (in Chinese)

\section{About the first author}

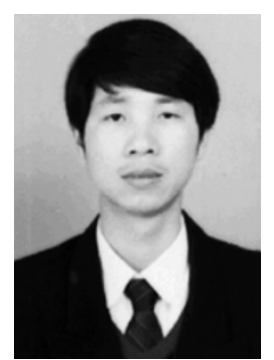

Ke Shizhen was born in 1967. He received his $\mathrm{PhD}$ in geophysical prospecting and information technology from the China University of Petroleum (Beijing) in 1999. Now he works at the China University of Petroleum with his interests in rock physical properties and geophysical well logging method and research on logging instruments. E-mail: wksz@vip.sina.com.

(Received July 16, 2006) (Edited by Zhu Xiuqin) 